\title{
Aircraft Landing Queue Scheduling Model on the Fight Delay Problem
}

\author{
Xin Zheng
}

Department of Electrical Engineering, North China Electric Power University, Baoding 07100, China 15733228927@163.com

Keywords: fight delay; aircraft landing queue scheduling; TSP; Hopfield neural network

\begin{abstract}
This paper aiming at the traffic control of fight to solve the problem of fight delay. To reinforce the traffic control, we build the aircraft queue scheduling model, which include two sub-models. When the amount of landing flights exceeds the runway capacity, the problem can transfer to the traveling salesman problem. Then we use Hopfield neural network to solve it to get the optimal aircraft queue.
\end{abstract}

\section{Introduction}

With the rapid change of economic and high-speed development of society, the civil aviation becomes more and more important in transportation system. Due to its rapid efficient transport capacity over a long distance, the civil aviation develops very fast. However, on the same time, Nowadays, the amount of flights delay and the average time of delay are also increasing fast in China. Among many influencing factors, airlines reason, traffic control, weather are major elements.

Considering degree of controllability, we build the aircraft queue scheduling model on traffic control to improve flight delay problem. In this paper, when the amount of landing aircrafts exceeds the runway capacity, it can transfer to the traveling salesman problem and use Hopfield neural model network to solve it. [1]

\section{The TSP Model}

If there are $n$ aircrafts arriving on the same time, they land one by one. Besides, different types of aircrafts must keep different safety time interval. Because of the difference of aircraft queue, the safety time intervals are different, too.

Our goal is to find the best aircraft queue to make sure the last aircraft land as soon as possible. The process is similar to TSP problem. [2]

We assume that there are $n$ aircrafts waiting to land, aircraft $i$ and aircraft $j$ keep safety time interval $T_{i j}$. When $x_{i j}=1, T_{i j}$ counts. So the objective function is as below.

$$
\min \sum_{i \neq j} T_{i j} x_{i j}
$$

Constraint condition is as below.

$$
x_{i j} \in\{0,1\}, i, j=1,2, \cdots, n, i \neq j
$$

Every point only has one line out, and has one line in.

$$
\begin{gathered}
\sum_{j=1}^{n} x_{i j}=1, i=1,2, \cdots, n \\
\sum_{i=1}^{n} x_{i j}=1, j=1,2, \cdots, n
\end{gathered}
$$

Besides, there are no points constitute a circle.

$$
\sum_{i, j \in s} x_{i j} \leq|s|-1,2 \leq|s| \leq n-1, s \subset\{1,2, \ldots, n\}
$$




\section{Hopfield Neural Network Model}

Hopfield neural network model reflects some characteristics of the human brain when it solves TSP problem. First, as for $n$ aircrafts, we use a $n \times n$ neurons matrix, which state of neuron shows one aircraft's location of time period. $v_{x i}$ shows the state of neurons, which $x, i \in$ $\{1,2, \cdots, n\}$. It means that aircraft $c_{x}$ is $i$ to land of particular time period. $v_{x i}=1$ means that $c_{x}$ appears at location $i$, and $v_{x i}=0$ means that $c_{x}$ doesn't appear at location $i$. So $n \times n$ neurons matrix $\boldsymbol{V}$ can determine all aircrafts' landing queue. To make sure every aircraft land only one time, so every row only has one element which is equal to 1 . Besides, on the certain time, there is only one aircraft landing, so every column only has one element which is equal to 1. [3]

Then it's turn to constitute a suitable energy function. Aiming at path validity, we have constraint condition as below.

$$
E_{1}=\frac{A}{2} \sum_{x=1}^{n} \sum_{i=1}^{n} \sum_{j=1, j \neq i}^{n} v_{x i} v_{x j}
$$

Which $A>0$ and $A$ is a constant. $E_{1}$ guarantees that $E_{1}$ reaches the minimum when $\boldsymbol{V}$ every row has no more than one element which is equal to 1 .

$$
E_{1 \min }=0
$$

In the same way, we have another constraint condition as below.

$$
E_{2}=\frac{B}{2} \sum_{x=1}^{n} \sum_{i=1}^{n} \sum_{j=1, j \neq i}^{n} v_{x i} v_{x j}
$$

Which $B>0$ and $B$ is a constant. $E_{2}$ guarantees that $E_{2}$ reaches the minimum when $\boldsymbol{V}$ every column has no more than one element which is equal to 1 .

Therefore we get the global constraint as below.

$$
E_{3}=\frac{C}{2}\left|\sum_{x=1}^{n} \sum_{i=1}^{n} v_{x i}-n\right|^{2}
$$

Which $C>0$ and $C$ is a constant. $E_{3}$ guarantees that $E_{3}$ reaches the minimum when $\boldsymbol{V}$ every row and column has no more than one element which is equal to 1 .

Considering the rationality of the time period, the energy function should contain the information of valid period.

$$
E_{4}=\frac{D}{2} \sum_{x}^{n} \sum_{y \neq x}^{n} \sum_{i}^{n} T_{x y} v_{x i}\left(v_{y, i+1}+v_{y, i-1}\right)
$$

Which $C>0$ and $C$ is a constant.

Therefore, the energy function is as below.

$$
E=E_{1}+E_{2}+E_{3}+E_{4}
$$

The admittance value between neurons $x_{i}$ and $y_{j}$ is as below.

$$
\begin{aligned}
& T_{x i, y j}=-A \delta_{x, y}\left(1-\delta_{i, j}\right)-B \delta_{i, j}\left(1-\delta_{x, y}\right)-C \\
& -D T_{x y}\left(\delta_{j, i+1}+\delta_{j, i-1}\right)\left(1-\delta_{x, y}\right)
\end{aligned}
$$

Which $\delta_{i, j}$ and $\delta_{x, y}$ can be defined as below.

And extrinsic motivation is as below.

$$
\delta_{i, j}= \begin{cases}1 & i=j \\ 0 & i \neq j\end{cases}
$$

$$
I_{x i}=C N
$$

To sum up, eventually dynamics equation is as below. 


$$
\begin{gathered}
C_{x i} \frac{d U_{x i}}{d t}=-\frac{U_{x i}}{R_{x i}} \\
-A \sum_{\substack{j \neq i \\
n}}^{n} v_{x j}-B \sum_{y \neq x}^{n} v_{x j}-C\left(\sum_{x}^{n} \sum_{y}^{n} v_{x y}-N\right) \\
-D \sum_{y \neq x} T_{x y}\left(v_{y, i+1}+v_{y, i-1}\right)
\end{gathered}
$$

\section{The Model Result}

If there are 8 aircrafts waiting to land, and they keep safety time interval, we use matlab to simulate 8 points to on behalf of 8 aircrafts. The first column represents aircrafts, and first row represents landing queue. The initial landing allocation is as below.

Tab.1 The initial landing queue

\begin{tabular}{lllllllll}
\hline & 1 & 2 & 3 & 4 & 5 & 6 & 7 & 8 \\
\hline 1 & 1 & & & & & & & \\
2 & & 1 & & & & & & \\
3 & & & 1 & & & & & \\
4 & & & & 1 & & & & \\
5 & & & & & 1 & & & \\
6 & & & & & & 1 & & \\
7 & & & & & & & & 1 \\
8 & & & & & & & \\
\hline
\end{tabular}

We use Hopfield neural network to solve TSP problem, then we get the optimal queue as below.

Tab.1 The optimal landing queue

\begin{tabular}{lllllllll}
\hline & 1 & 2 & 3 & 4 & 5 & 6 & 7 & 8 \\
\hline 1 & & & & & & & 1 & \\
2 & & & & 1 & & & & \\
3 & & & & & 1 & & & \\
4 & 1 & & & & & & & \\
5 & & & 1 & & & & & \\
6 & & 1 & & & & & & 1 \\
7 & & & & & & 1 & & \\
8 & & & & & &
\end{tabular}

So the queue is $4 \rightarrow 6 \rightarrow 5 \rightarrow 2 \rightarrow 3 \rightarrow 8 \rightarrow 1 \rightarrow 7 \rightarrow 4$.

Matlab draw the initial and the optimal landing queue is as below.
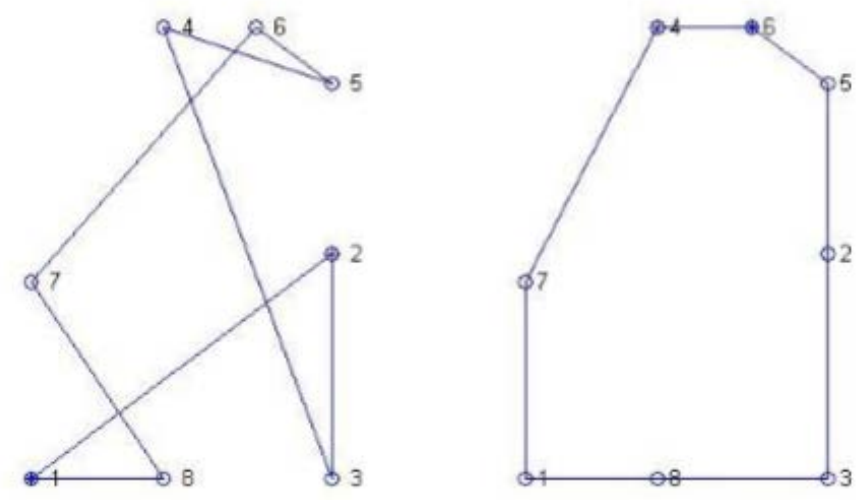

Fig.1 The initial landing queue (left) and the optimal landing queue (right) 


\section{Summary}

Through transferring the aircraft landing queue problem to TSP problem, then use Hopfield neural network to solve it, we can get the optimal queue. The shortest path corresponds to the shortest time of aircraft landing queue. So the model can effectively reduce the rate of flight delays, and it contributes to traffic control.

\section{References}

[1] Liang Wu. The Study of Aircraft Sequencing in the Terminal Area Airport Issue [J]. Shanghai: Shanghai Jiao Tong University, 2005, 15.

[2] Shoukui Si, Xijing Sun. Mathematical Modeling Algorithm and Application. National Defence Industry Press, 2011.

[3] Jun Fan, Lili Wang. The Single Airport Ground-holding Optimization Algorithm Based on the Hopfield Network [J]. China's Civil Aviation College Journals, 2004, 7(2):11-13.

[4] http://www.flightstats.com 